Supporting information

\title{
InterMetalDB: a Database and Browser of \\ Intermolecular Metal Binding Sites in Macromolecules \\ with Structural Information
}

Józef Ba Tran ${ }^{a}$ and Artur Krężel*a

\begin{abstract}
Contribution from
${ }^{a}$ Department of Chemical Biology, Faculty of Biotechnology, University of Wroctaw, F. Joliot-Curie 14a, 50-383 Wrocław, Poland
\end{abstract}




\section{Table of contents}

Figure S1. Number of donors in a metal site depending on a lanthanide metal ion. Graphs are prepared for a representative dataset.

Figure S2. Number of donors in a metal site depending on a metal ion. Graphs are prepared for a non-representative dataset.

S4

Figure S3 Number of donors in a metal site depending on a lanthanide metal ion. Graphs are prepared for a non-representative dataset. . $\mathrm{S} 5$

Figure S4. Number of chains creating metal sites, depending on a bound metal ion. Graphs are prepared for a representative dataset.

Figure S5. Number of chains creating metal sites, depending on a bound metal ion. Graphs are prepared for a non-representative dataset. .S7

Figure S6. Number of chains creating metal sites, depending on a bound lanthanide metal ion. Graphs are prepared for a non-representative dataset.... .S8 

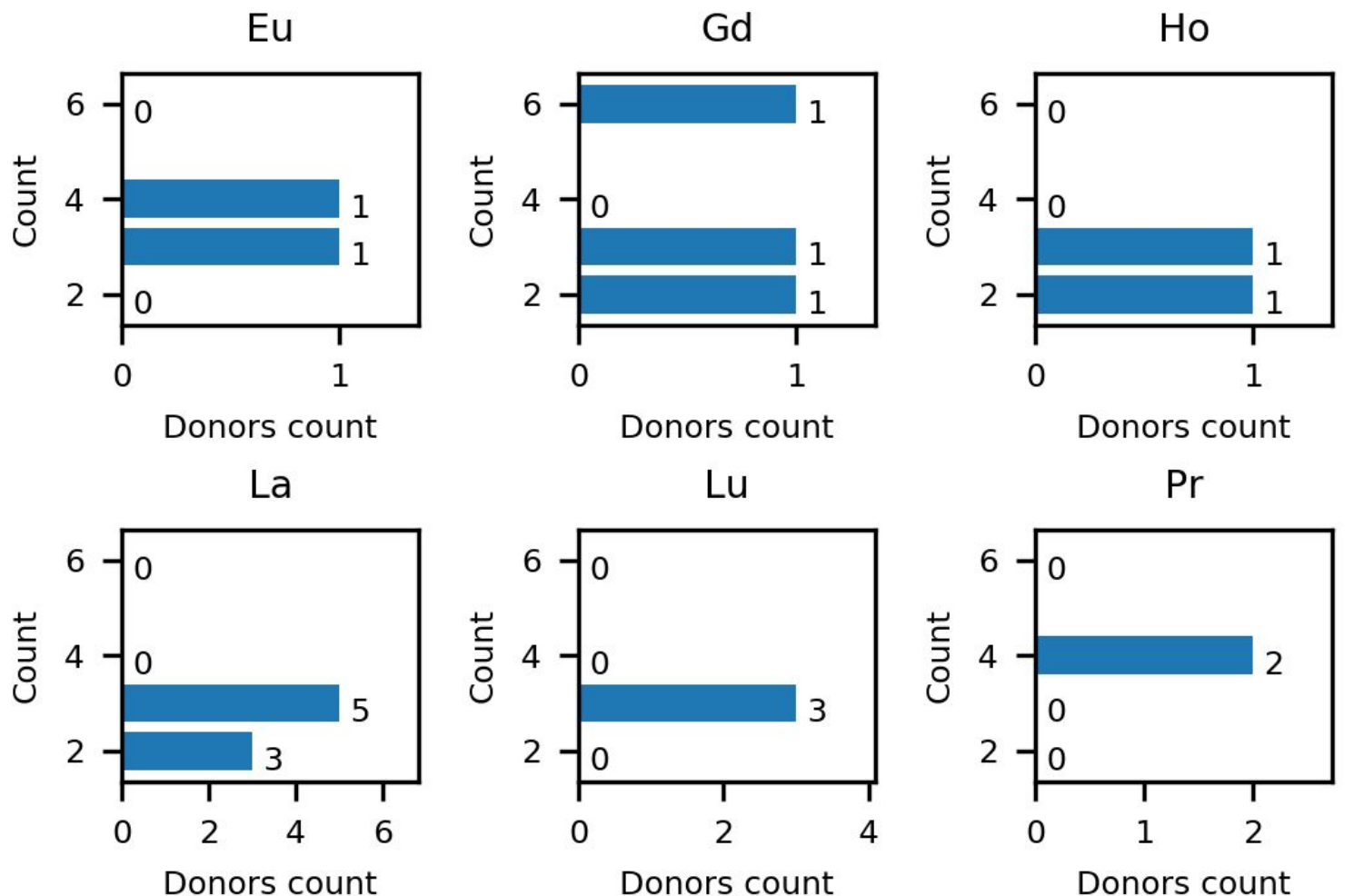

$\mathrm{Lu}$
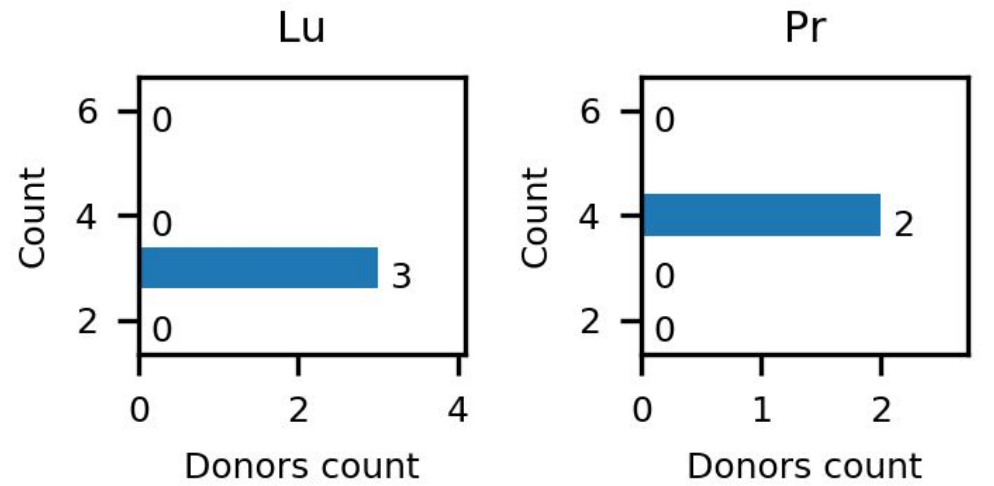

$\mathrm{Sm}$

$\mathrm{Tb}$

$\mathrm{Yb}$
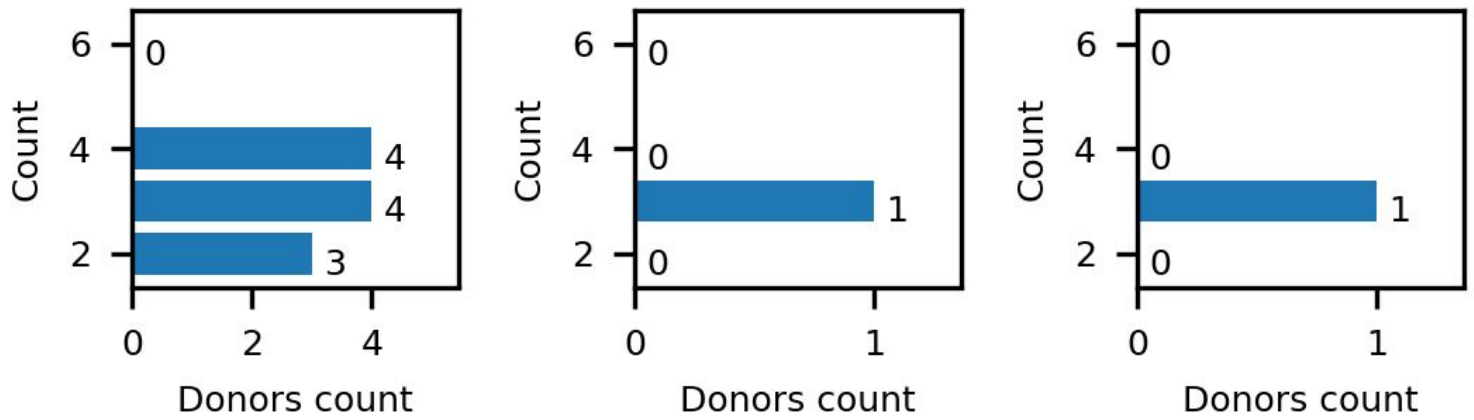

Figure S1. Number of donors in a metal site depending on a lanthanide metal ion. Graphs are prepared for a representative dataset. 

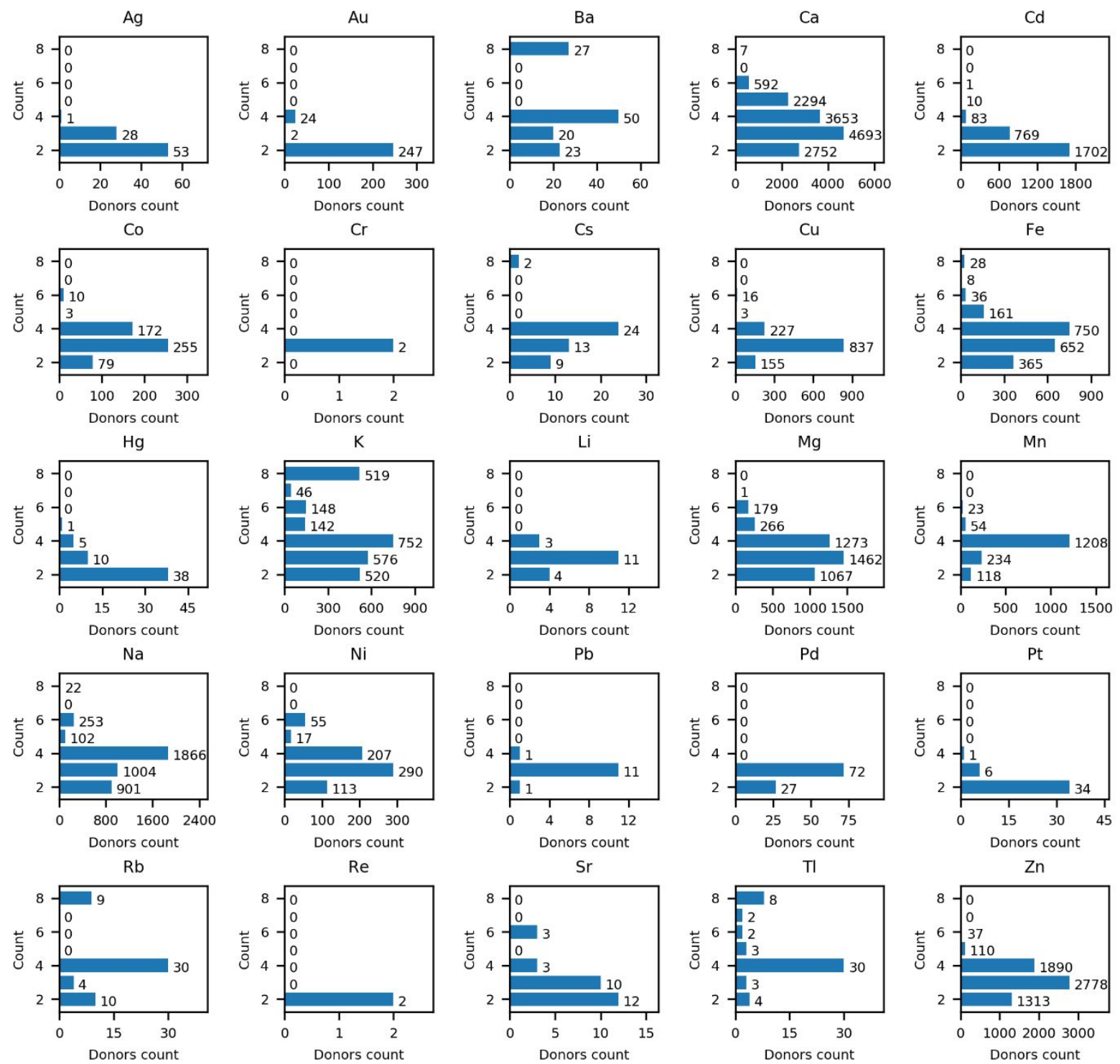

Figure S2. Number of donors in a metal site depending on a metal ion. Graphs are prepared for a non-representative dataset. 

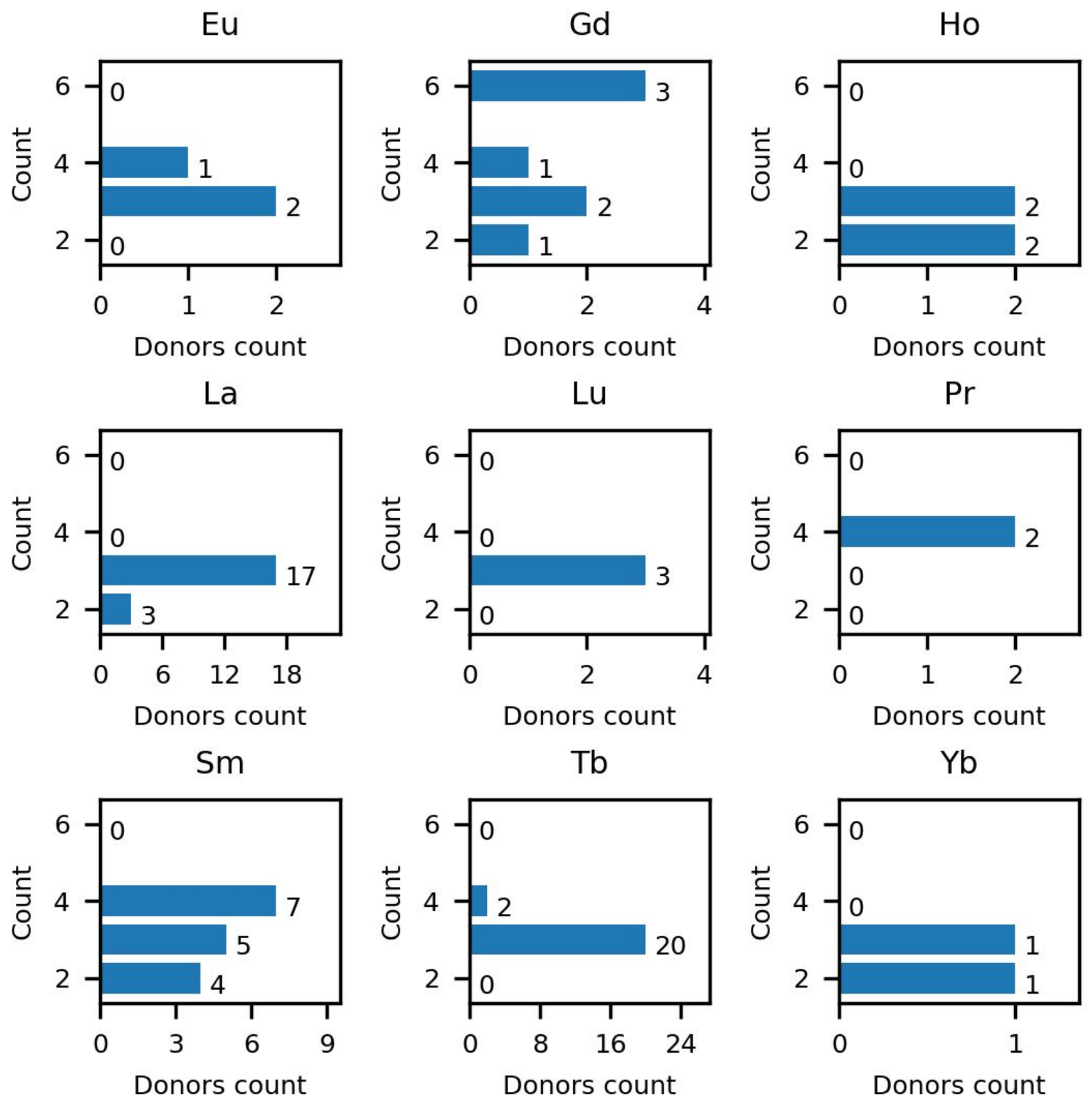

$\mathrm{Tb}$
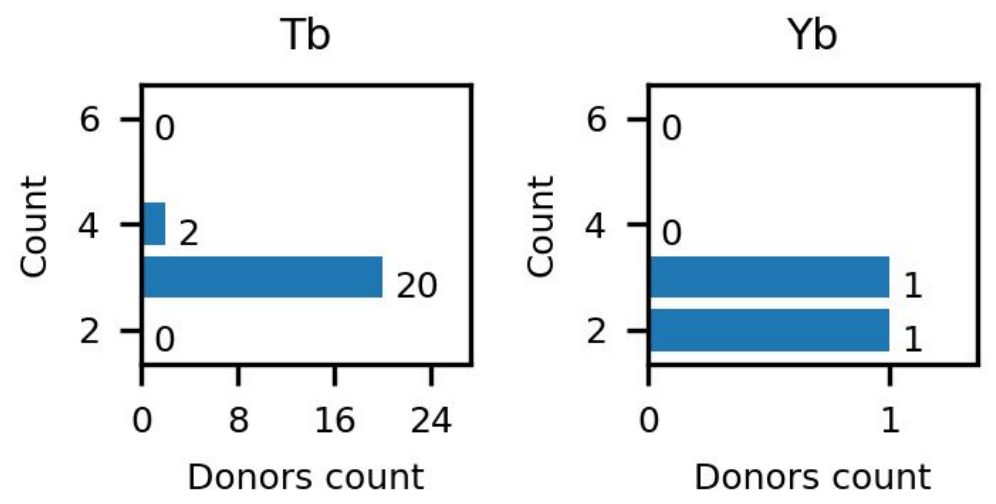

Figure S3. Number of donors in a metal site depending on a lanthanide metal ion. Graphs are prepared for a non-representative dataset. 


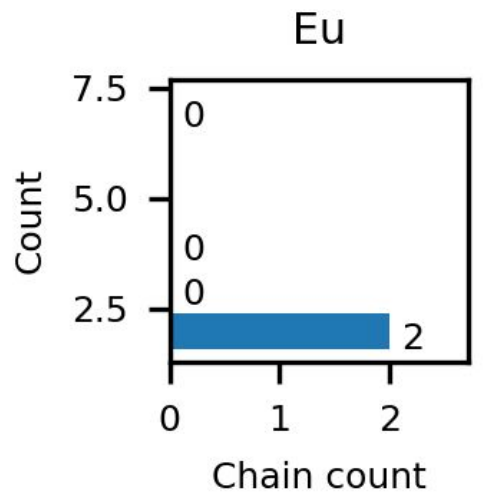

La

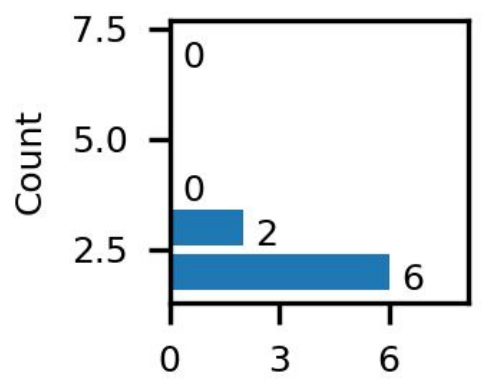

Chain count

$\mathrm{Sm}$

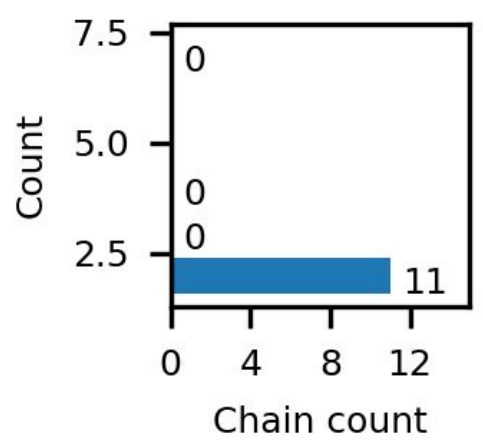

Gd

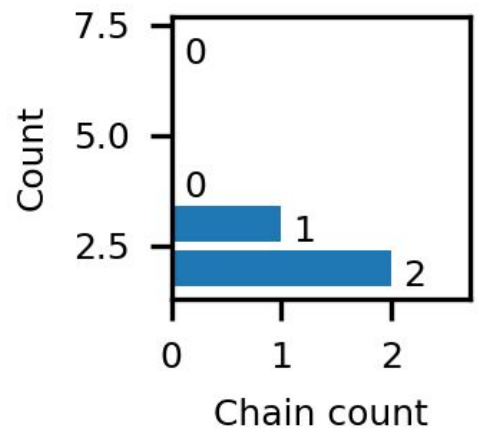

Lu

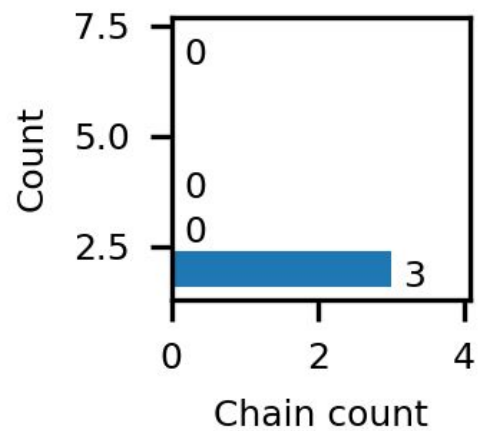

$\mathrm{Tb}$

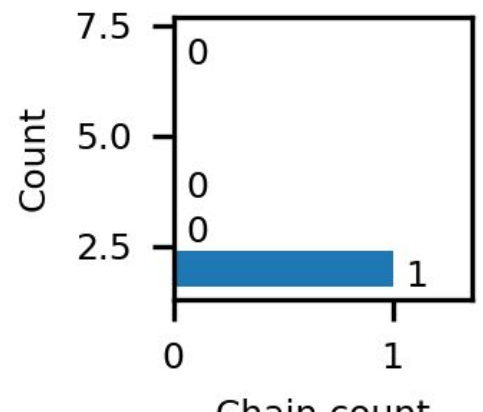

Ho

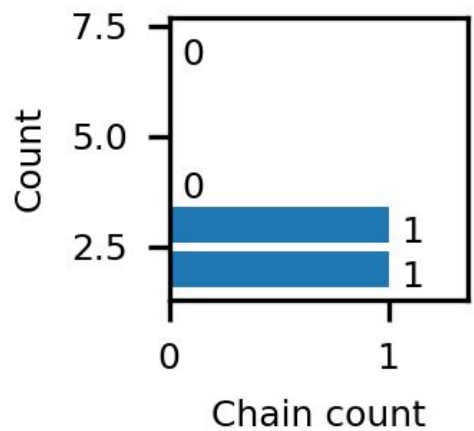

$\mathrm{Pr}$

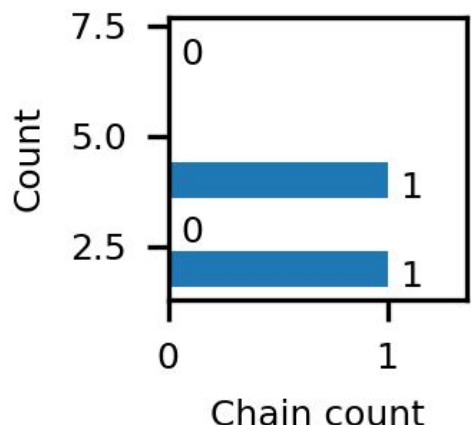

$\mathrm{Yb}$

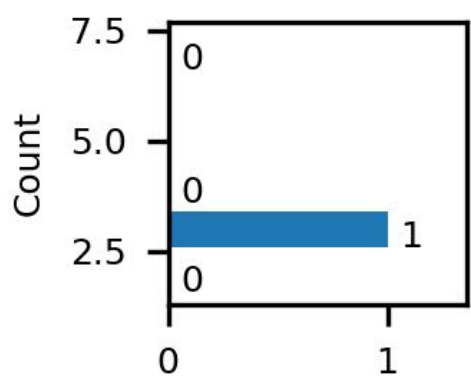

Chain count

Figure S4. Number of chains creating metal sites, depending on a bound metal ion. Graphs are prepared for a representative dataset. 


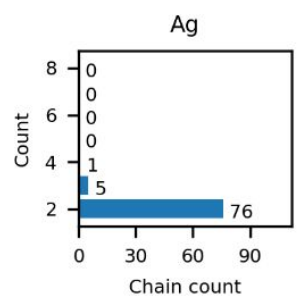

Co

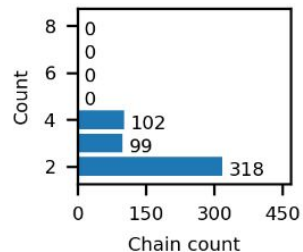

$\mathrm{Hg}$

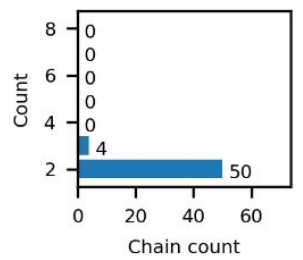

$\mathrm{Na}$

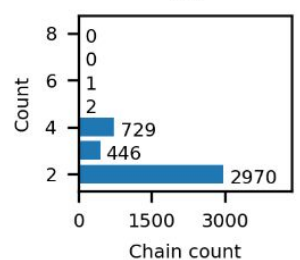

$\mathrm{Rb}$

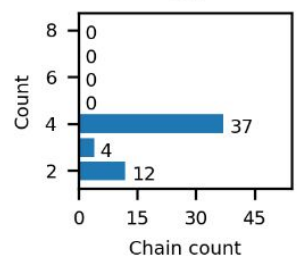

$\mathrm{Au}$

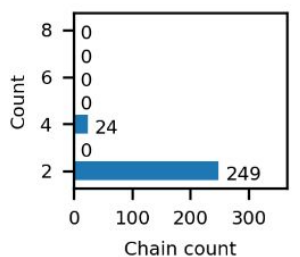

$\mathrm{Cr}$

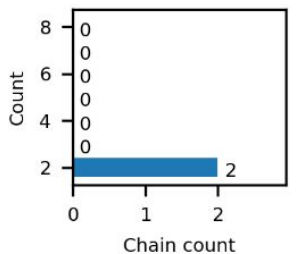

K

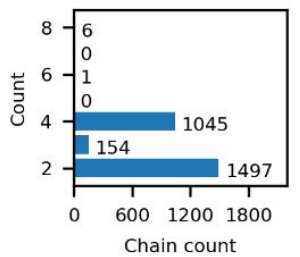

$\mathrm{Ni}$

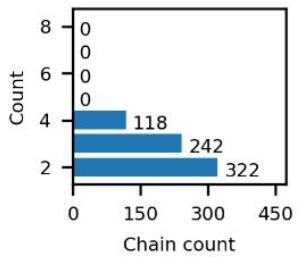

$\mathrm{Re}$

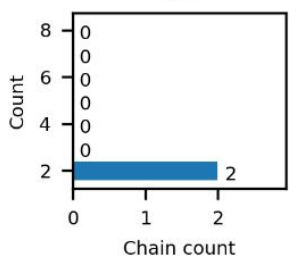

Ba

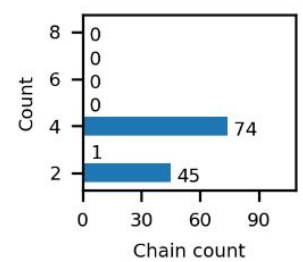

Cs

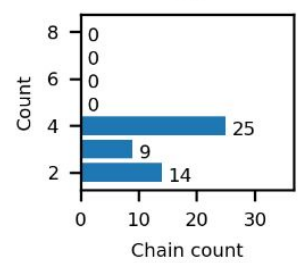

Li

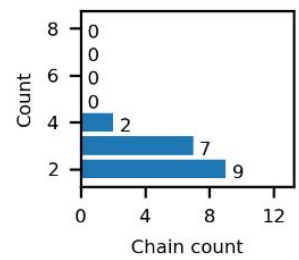

$\mathrm{Pb}$

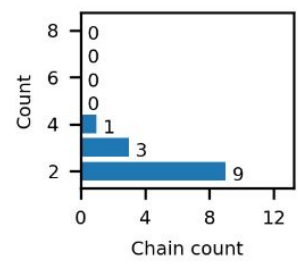

$\mathrm{Sr}$

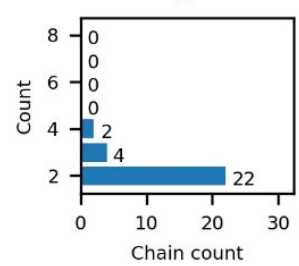

Ca

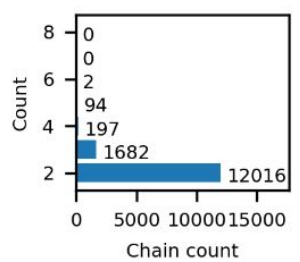

$\mathrm{Cu}$

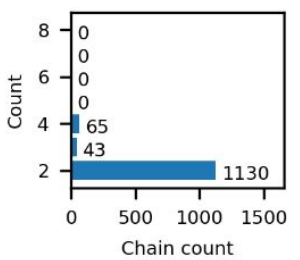

$\mathrm{Mg}$

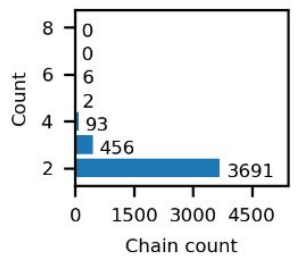

$\mathrm{Pd}$

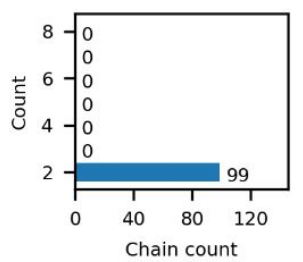

$\mathrm{TI}$

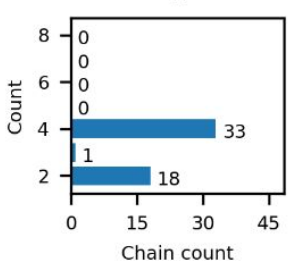

$\mathrm{cd}$

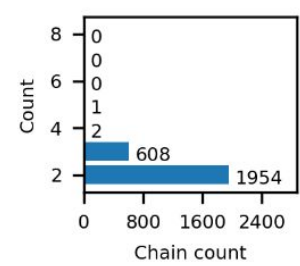

$\mathrm{Fe}$

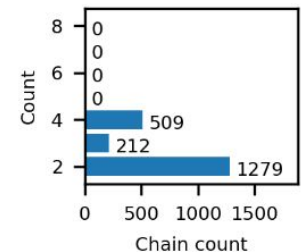

$\mathrm{Mn}$

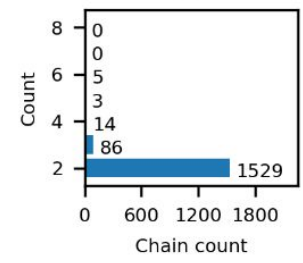

Pt

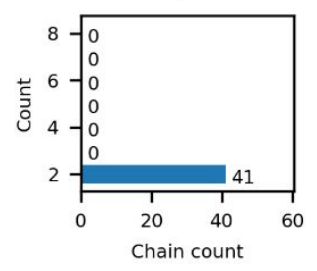

$\mathrm{Zn}$

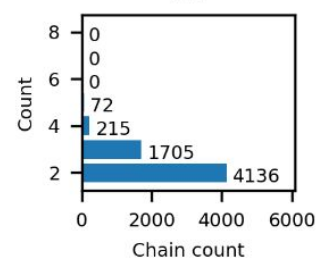

Figure S5. Number of chains creating metal sites, depending on a bound metal ion. Graphs are prepared for a non-representative dataset. 

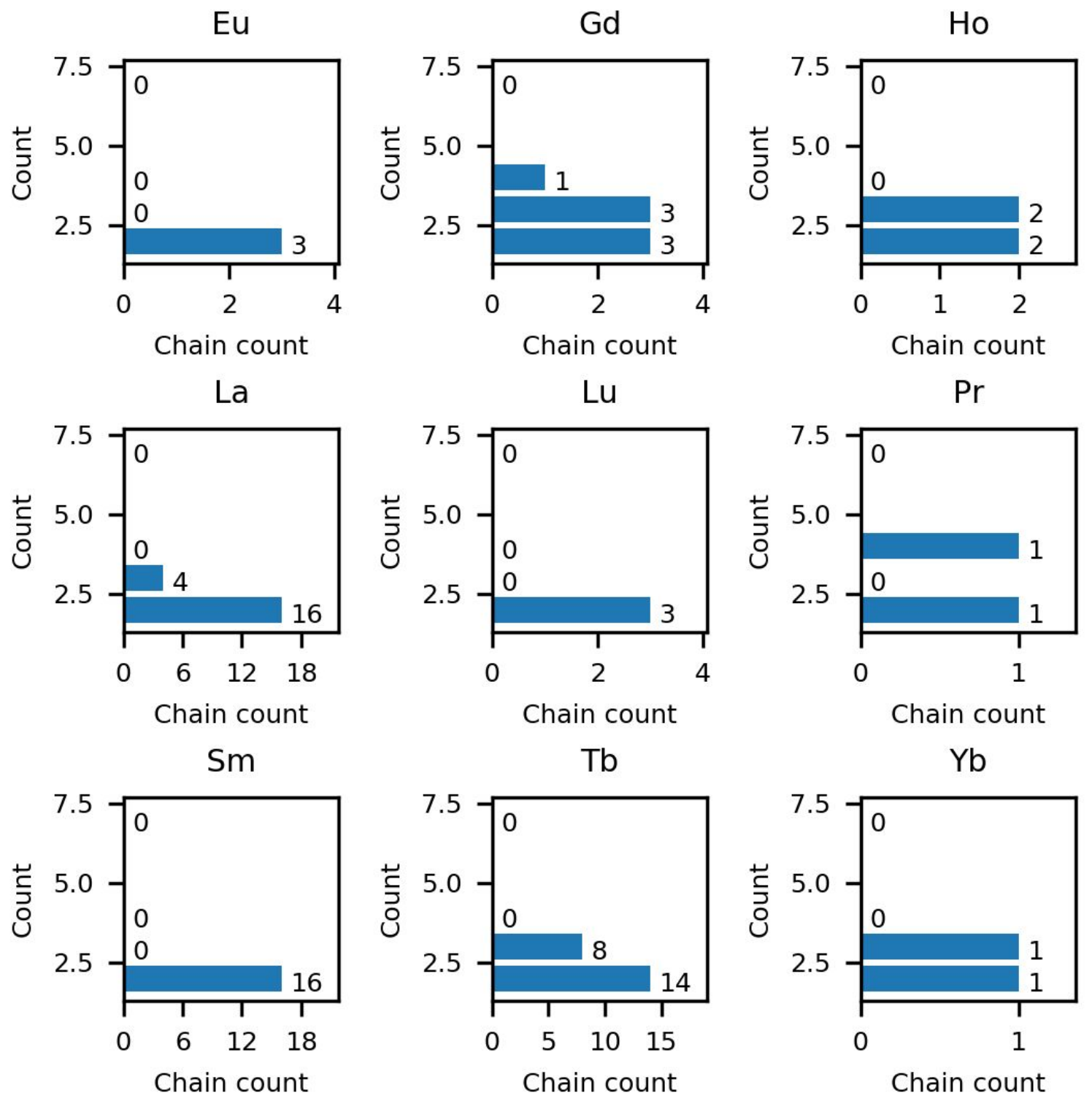

Figure S6. Number of chains creating metal sites, depending on a bound lanthanide metal ion. Graphs are prepared for a non-representative dataset. 